\title{
COMPARING THE EFFECT OF PROBIOTIC STREPTOCOCCUS SALIVARIUS K12 AND M18 ON THE STREPTOCOCCUS MUTANS COUNT, SALIVARY PH AND BUFFER CAPACITY: A RANDOMIZED DOUBLE BLINDED CLINICAL TRIAL
}

\begin{abstract}
Aim: There is a growing interest in the use of beneficial bacteria such as probiotics to modulate the oral microbiota. Literature reveals a number of studies on the key species Streptococcus salivarius K12 and M18 on prevention of dental caries. However, there is a paucity in the clinical studies on the effect of salivarius $\mathrm{K} 12$ on $S$. mutans count. In addition to this, the effect of salivarius K12 and M18 on the salivary $\mathrm{pH}$ and buffering capacity have also not been studied. Thus the aim of the present study was to evaluate and compare the effect of probiotic Streptococcus salivarius K12 and M18 on the Streptococcus mutans count, salivary $\mathrm{pH}$ and buffer capacity.
\end{abstract}

Materials and method: 146 subjects were screened for eligibility and 69 Subjects within the age group of 18-40 years were randomly allocated to three groups of 23 subjects each. Subjects enrolled in Group A received BLIS $\mathrm{K} 12^{\mathrm{TM}}$, Subjects in Group B received BLIS M18 ${ }^{\mathrm{TM}}$ and Subjects in Group C belonged to the control group and did not receive any form of probiotics. Unstimulated salivary samples were collected at baseline and after 30 days. The samples were analysed for Streptococcus mutans level, salivary pH and buffer capacity.

Results: Among the 69 subjects enrolled for the study, 6 subjects were lost to follow up and 63 subjects completed the trial. A statistically significant reduction in salivary $S$. mutans levels $(\mathrm{p}=0.001)$ and an increase in the salivary $\mathrm{pH}(\mathrm{p}=0.001)$ was observed after the use of probiotics when compared to the baseline. The buffer capacity remained unaltered following the use of both the probiotics. There was no change in the $S$. mutans count $(\mathrm{p}=0.065)$, salivary $\mathrm{pH}$ values $(\mathrm{p}$ value $=0.242)$ and buffer capacity $(\mathrm{p}=0.87)$ for the subjects belonging to the control group.

Conclusions: Within the limitations of the present study it can be concluded that a 30day use of Streptococcus salivarius K12 and M18 resulted in a reduction in the Streptococcus mutans count while simultaneously improving the salivary $\mathrm{pH}$.

Keywords: Dental caries, Oral health, Probiotics, Streptococcus salivarius.
*Saravanan Poorni ${ }^{1}$

Nivedhitha $\mathrm{MS}^{2}$

Manali Ramakrishnan

Srinivasan $^{1}$

ORCID IDs of the authors:

S.P. $\quad 0000-0001-8811-2960$

N.M.S. $\quad$ 0000-0001-8979-6719

M.R.S. 0000-0002-3651-181X
Received : 24.05.2021

Accepted : 03.11 .2021

\footnotetext{
How to Cite Poorni S, MS N, Ramakrishnan Srinivasan M. Comparing The Effect of Probiotic Streptococcus Salivarius K12 and M18 on the Streptococcus Mutans Count, Salivary pH And Buffer Capacity: A Randomized Double Blinded Clinical Trial. Cumhuriyet Dent J 2021;24:4:346-354.

*Corresponding Author:

Department of Conservative Dentistry and Endodontics, Sri Venkateswara Dental College and Hospital, Off OMR road, Thalambur, Kanchipuram District - 603103. Phone: 0091-4427435059 Mobile Phone: 0091-9941123738 Fax: 0091-4427435059 E-mail: $161703003 . s d c @$ saveetha.com
} 


\section{INTRODUCTION}

Dental plaque is a biofilm composed of various microbial species and their products, is attributed as a risk factor of oral infectious diseases such as periodontitis and dental caries. ${ }^{1-3}$ Current literature attributes oral diseases to a complex interactions between virulent microorganisms, their products, frequency of carbohydrates consumption and also the host salivary composition. ${ }^{4-6}$ Among the various pathogens studied, Streptococcus mutans (S. mutans) plays a vital role in pathogenesis of dental caries. ${ }^{4,7}$ Whilst being only weakly competitive at neutral $\mathrm{pH}$, this cariogenic bacteria dominates the dental biofilm under acidic conditions caused by catabolism of dietary carbohydrates. ${ }^{8}$ With recent interest on the role of ecological imbalance and dental caries pathogeneisis, there is a growing interest in the use of beneficial bacteria such as probiotics thereby modulating the oral microbiota. ${ }^{9-11}$

Probiotics are defined as those "Live microorganisms which when administered in adequate amounts confer a health benefit to the host". ${ }^{12}$ They are expected to accomplish certain functional requirements such as resistance to acid, adherence to epithelial surfaces and inhibitory activity against some pathogens. ${ }^{13}$ Though most probiotics are gram positive bacteria, they belong to the genera Lactobacillus and Bifidobacterium. ${ }^{14}$ However due to their limitations in terms of colonisation of oral tissues, a new generation of probiotic strains, Streptococcus salivarius, known to have low pathogenic potential is currently being extensively studied. ${ }^{14-17}$

Literature highlights a number of studies on the key species Streptococcus salivarius K12 and M18 on prevention of dental caries. Recent studies have revealed along with the safety and tolerability profiles, the capacity of this genera to reduce plaque formation and also to lower $S$. mutans counts in the oral cavity. ${ }^{15,17}$ Pierro et al. ${ }^{15}$ in his study demonstrated that in children who are at high risk for developing caries, when treated with a probiotic containing Streptococcus salivarius M18, they are less likely to develop dental caries. Burton et al. ${ }^{17}$ in his study confirmed that Streptococcus salivarius M18 demonstrated a greater plaque reduction and a higher reduction in $S$. mutans count when compared to those subjects who were merely exposed to bacterial probiotics.

However, there is a paucity in the clinical studies on the effect of salivarius $\mathrm{K} 12$ on $S$. mutans count. In addition to this, the effect of salivarius $\mathrm{K} 12$ and M18 on the salivary $\mathrm{pH}$ and buffering capacity have also not been studied. Thus the current trial was undertaken to evaluate and compare the effect of probiotic Streptococcus salivarius $\mathrm{K} 12$ and M18 on the Streptococcus mutans count, salivary $\mathrm{pH}$ and buffer capacity.

\section{MATERIALS AND METHODS}

\section{Study Design:}

The objective of this study was to assess the variation in the Streptococcus mutans count, salivary $\mathrm{pH}$ and buffer capacity following the use of probiotic lozenges containing strains Streptococcus salivarius K12 and Streptococcus salivarius M18. The double blinded placebo controlled clinical trial adhered to the CONSORT statement and was conducted in the Department of Conservative Dentistry and Endodontics, Sri Venkateswara Dental College and Hospital, Chennai from December 2019 for a period of 30 days. This study was conducted in accordance with the 1964 Declaration of Helsinki and its subsequent amendments. Ethical clearance was acquired from the institutional ethical committee and informed consent was taken from all the participants enrolled in the current trial.

\section{Subjects and Criteria:}

7 days prior to the commencement of the 30-day trial, 146 subjects were screened for eligibility. Based on the inclusion and exclusion criteria, 69 participants were enrolled for the trial. Subjects in the age group of 18-40 years, with a DMFT score of 4 and above were included in the trial. Subjects with systemic conditions, periodontal diseases, individuals who were on antibiotics in the last 2 weeks, individuals who had allergic history, pregnant and lactating women were excluded from the trial. During the trial period, subjects were instructed not to take probiotics in other forms for any other reason. A pilot study was conducted and based on the 
S.mutans count in the pilot study, sample size was calculated using $G$ Power Analysis (version 3.1.9.7). A power calculation indicated that 60 subjects were required to provide a $90 \%$ chance of detecting the effect size in a continuous outcome measure, assuming a significance level of $5 \%$. The $\beta$ error was calculated at 0.10 . Since an attrition rate of $15 \%$ was expected, a total of 69 subjects were enrolled for the study.

\section{Grouping of Subjects and Randomisation:}

Streptococcus salivarius that were marketed as probiotic lozenges in two forms as Teeth and Gums BLIS K12 ${ }^{\mathrm{TM}}$ and Throat Health BLIS $\mathrm{M} 18^{\mathrm{TM}}$ were used for the trial. According to the protocol, 69 subjects both male and female were recruited and randomly allocated to 3 groups of 23 subjects each. Subjects enrolled in Group A received BLIS $\mathrm{K} 12^{\mathrm{TM}}$, Subjects in Group B received BLIS M18 ${ }^{\mathrm{TM}}$ and Subjects in Group C belonged to the control group and did not receive any form of probiotics.

The subjects enrolled for the trial were allocated into 3 groups with the aid of a simple randomisation method. For allocation, a computer generated list of random numbers was used with the ratio of randomisation of $1: 1: 1$ by using random allocation software (Version 2.0). Allocation sequences were concealed from the researchers who were a part of the study in order to reduce the selection bias.

\section{Treatment Protocol:}

Subjects enrolled in the 2 test groups that is Group A and Group B were instructed to take one lozenge of BLIS $\mathrm{K} 12^{\mathrm{TM}}$ or BLIS M18 ${ }^{\mathrm{TM}}$ for 30 consecutive days based on the group to which they belonged to. Subjects were instructed to slowly dissolve one lozenge on the tongue during bedtime, ideally after brushing. All subjects were asked to follow the regular oral hygiene procedures that were followed routinely. Subjects were also instructed not to eat or drink for at least 30 minutes' post ingestion. In order to evaluate the level of adherence to treatment protocol, the subjects were asked to return any unused product boxes and tablets. Acceptable adherence was considered to be administration of not less than $95 \%$ of the lozenges allocated. Subjects belonging to the control group (Group C) did not receive any treatment and were asked to followonly the regular oral hygiene procedures.

\section{Streptococcus mutans count, Salivary pH and Buffer Capacity Testing:}

In order to obtain the samples for analysis of $S$. mutans, salivary $\mathrm{pH}$ and buffer capacity, Unstimulated whole saliva was collected from all the subjects enrolled for the study. The subjects were asked to refrain from talking and drop their head down so as to let the saliva run naturally to the front of the mouth. The subjects were also instructed not to cough up mucus as saliva is collected. They were made to spit into sterile bottles about once in a minute for up to 5 minutes until 3-4 millilitre of unstimulated saliva was collected. Samples were collected twice. Once at baseline that is before the start of the study and the second time after 30 days' use of probiotic lozenges. A pH meter (Digital pH meter, MIFA Systems Private Limited, Ahmedabad, Gujarat, India) was used to record the $\mathrm{pH}$ of the unstimulated saliva samples. About $0.1 \mathrm{ml}$ of the sample was taken, diluted and vortex mixed. 0.1 $\mathrm{ml}$ of this diluted sample was then taken to inoculate onto the Mitis Salivarius Agar base (HIMEDIA, Mumbai, Maharashtra, India). This followed by incubating the petri dishes at $37^{\circ} \mathrm{C}$ with $3 \% \mathrm{CO}_{2}$ for 48 hours. The organisms were identified based on their colony morphology. The colony forming units were counted manually. Buffer capacity was evaluated using a handheld $\mathrm{pH}$ meter. Initially the $\mathrm{pH}$ sensitive electrode was calibrated for $\mathrm{pH} 4.0$ and 7.0 using standard $\mathrm{pH}$ pellets. Two hundred and fifty microliters of lactic acid $(\mathrm{pH} \mathrm{3}, 1.5 \mathrm{mM})$ were titrated into the test sample and mixed. $\mathrm{pH}$ value for the titrated sample was then noted using the handheld $\mathrm{pH}$ meter with digital reading display. The results were ranked as high $(>5.8)$, medium $(<5.7$ and $>4.8)$ or low $(\mathrm{pH}<4.7)$.

\section{Statistical Analysis}

Data obtained at baseline and 30 days were recorded using Microsoft Office Excel 2016 and subjected to statistical analysis using SPSS Software version 22. Chi-square test was used to analyse the statistical difference in terms of Age 
and sex among the subjects enrolled in the three group. Data was analysed for normal distribution of variables using One sample KolmogorovSmirnov test. The variables were found to be normally distributed for $S$. mutans count and salivary $\mathrm{pH}$. Hence paired t- test was used for within the group comparisons and one-way ANOVA was used for inter group comparisons. Data on buffer capacity being ordinal in nature, Wilcoxon- signed rank test was used for within the group comparison and Kruskal Wallis test was used for inter group comparisons. The level of statistical significance was set at 5\% $(\mathrm{p}<0.05)$.

\section{RESULTS}

This randomised controlled clinical trial has been carried out on 63 subjects. Though 69 subjects were enrolled for the study, 6 subjects were lost to follow. Figure 1 shows the total number of subjects enrolled for the study and the number of subjects in each group who completed the trial with details of the subjects excluded from the trial.

Figure: Flow Diagram showing information on excluded patients

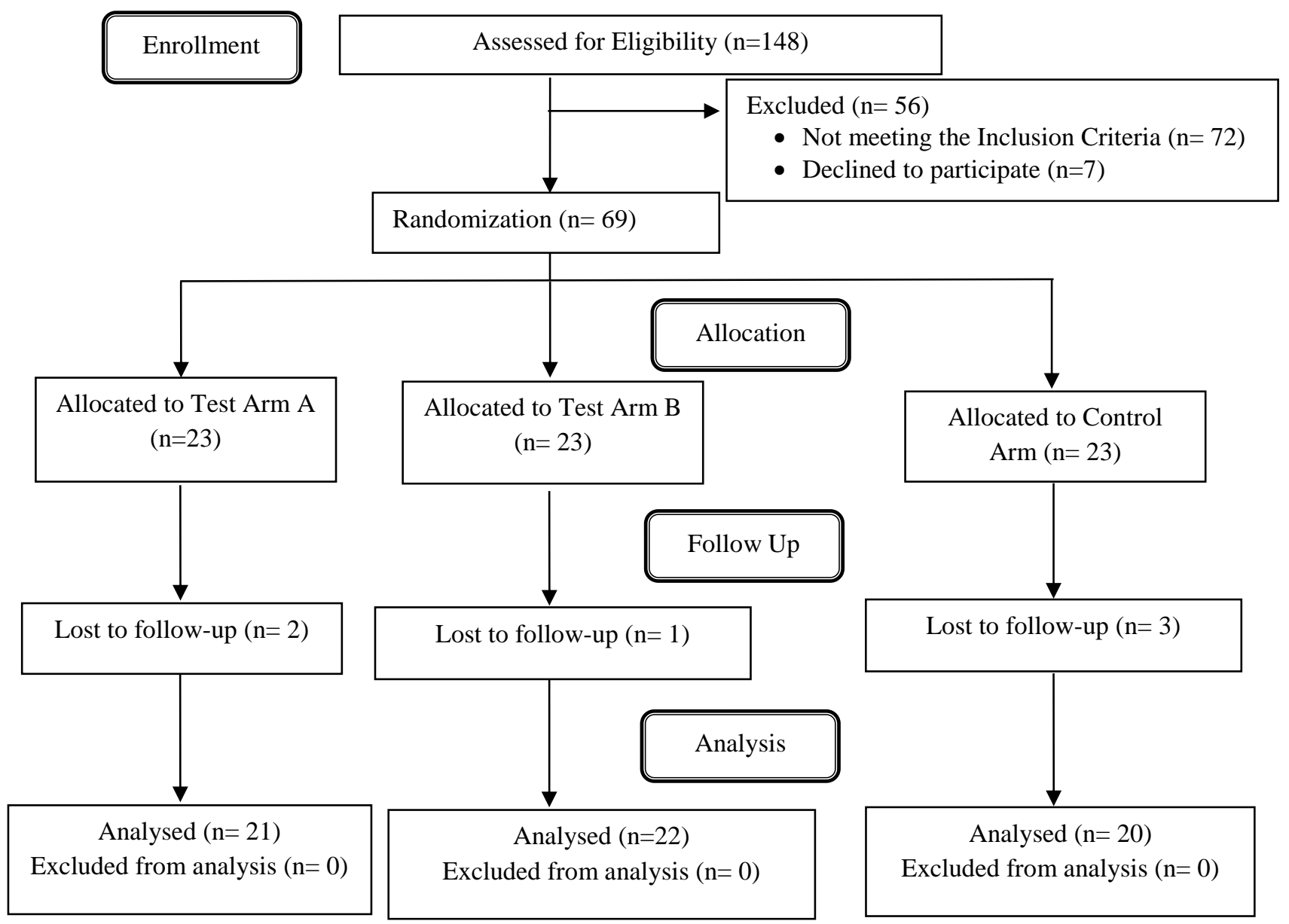

Among the 63 subjects who completed the trial, 38 were males and 25 were females. The mean age of the subjects enrolled for the trial was $26.01 \pm 5.78$ yrs. The mean DMFT score of subjects enrolled for the study was 5.9. As shown in Table 1, no statistical difference was observed between the three groups in terms of sex $(\mathrm{p}=0.739)$, age $(\mathrm{p}=0.072)$ and DMFT score $(\mathrm{p}=0.06)$. The dropout rate from the baseline was low and the compliance with the study protocol was found to be satisfactory. 
Table 1: Mean age and Distribution of the study population based on their gender among the 3 groups

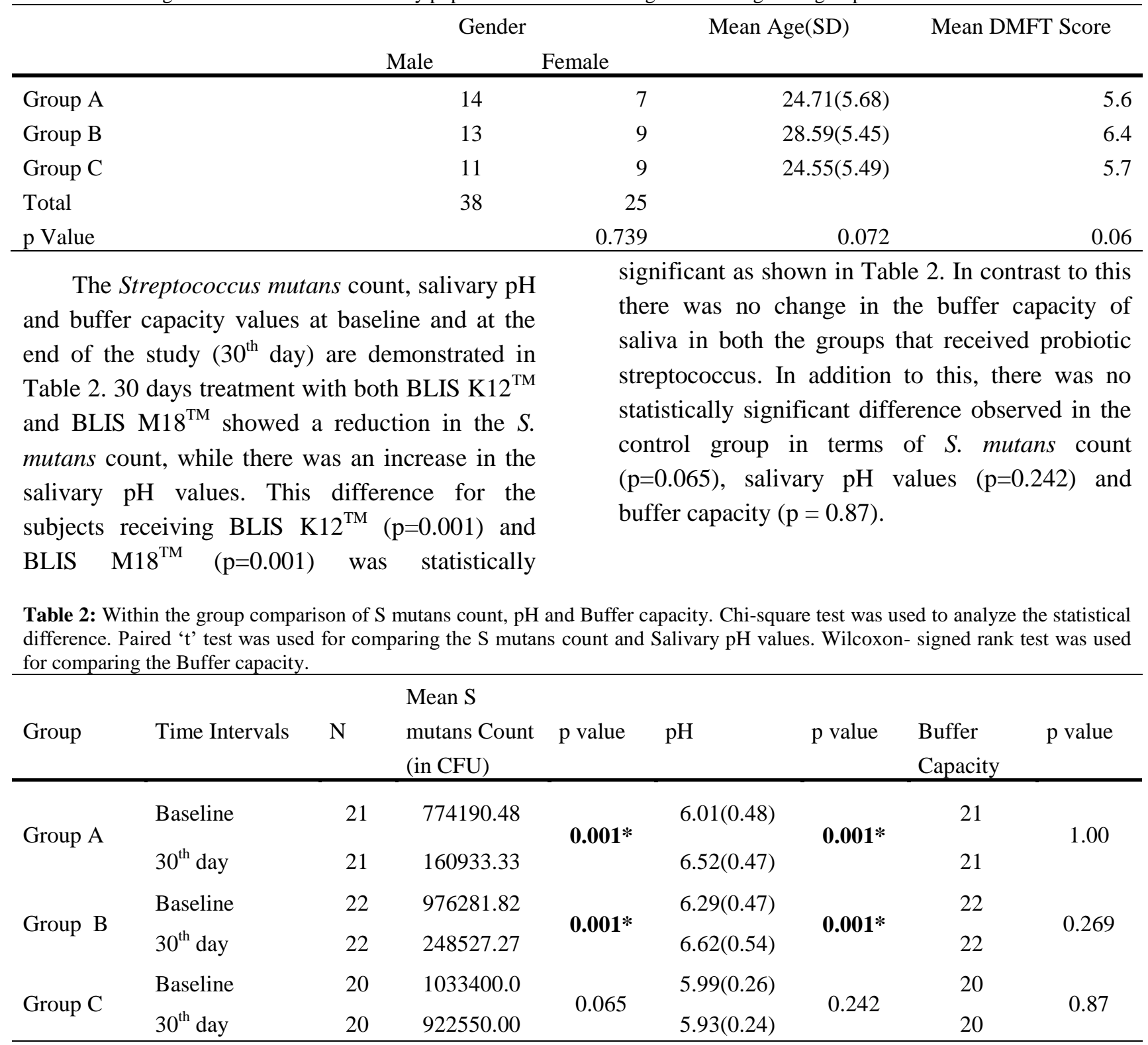

Table 3 shows comparison of $\mathrm{S}$ mutans count, $\mathrm{pH}$ and Buffer capacity among the three groups. Though there was no significant difference at baseline in the $S$. mutans count $(\mathrm{p}=$ $0.76), \mathrm{pH}(\mathrm{p}=0.057)$ and buffer capacity ( $\mathrm{p}$ value $=0.872$, while analysis of data obtained after 30days showed that the number of $S$. mutans colonies were found to be higher in the control group than the two test groups and this difference was found to be statistically significant $(\mathrm{p}=0.001)$. However, at the 30th day, there was no significant difference between the $S$. mutans count and salivary $\mathrm{pH}$ levels obtained from the subjects enrolled in the two probiotic groups $(\mathrm{p}=0.607)$. 
Table 3: Comparison of $\mathrm{S}$ mutans count, $\mathrm{pH}$ and Buffer capacity among the three groups at baseline and after 30 days. One Way ANOVA followed by Post Hoc Tukey test was used for comparing the S mutans count and Salivary pH values. Kruskal Wallis test was used for comparing the Buffer capacity.

\begin{tabular}{|c|c|c|c|c|c|c|c|c|}
\hline \multirow{2}{*}{$\begin{array}{l}\text { Time } \\
\text { Intervals }\end{array}$} & \multicolumn{4}{|c|}{ Mean S mutans } & \multirow[b]{2}{*}{$\mathrm{pH}$} & \multirow[b]{2}{*}{$\mathrm{p}$ value } & \multirow{2}{*}{$\begin{array}{c}\text { Buffer } \\
\text { Capacity }\end{array}$} & \multirow[b]{2}{*}{$\mathrm{p}$ value } \\
\hline & & $\mathrm{N}$ & $\begin{array}{c}\text { Count } \\
\text { (in } \mathrm{CFU}) \\
\end{array}$ & $\mathrm{p}$ value & & & & \\
\hline \multirow{3}{*}{ Baseline } & Group A & 21 & 774190.48 & & $6.01(0.48)$ & & 21 & \multirow{3}{*}{0.872} \\
\hline & Group B & 22 & 976281.82 & 0.76 & $6.29(0.47)$ & 0.057 & 22 & \\
\hline & Group C & 20 & 1033400.00 & & $5.99(0.26)$ & & 20 & \\
\hline \multirow{3}{*}{$30^{\mathrm{TH}}$ Day } & Group A & 21 & 160933.33 & & $6.52(0.47)$ & & 21 & \multirow{3}{*}{$0.001 *$} \\
\hline & Group B & 22 & 248517.27 & $0.001 *$ & $6.62(0.54)$ & $0.001 *$ & 22 & \\
\hline & Group C & 20 & 922550.00 & & $5.93(0.24)$ & & 20 & \\
\hline
\end{tabular}

\section{DISCUSSION}

Streptococcus mutans has been associated with dental caries due to its ability to metabolize the sugar substrates to produce acids that demineralizes the tooth structure. ${ }^{18}$ Thus an increase in the $S$. mutans level in the saliva favours demineralisation. ${ }^{19,20}$ Enhancing the salivary defence mechanism while diminishing the microbial levels seems to be a rational approach. Salivary $\mathrm{pH}$ and buffer are two salivary defence mechanisms that prevent demineralisation. ${ }^{21}$ In the current era, focus has always been on caries prevention which is researched at large. There has always been a constant surge to identify preventive measures for dental caries. Thus evaluating the reduction of Streptococcus mutans counts along with change in salivary $\mathrm{pH}$ and buffer capacity is deemed necessary to establish the caries preventive effect for any intervention. Thus the current trial evaluated the impact of the probiotic strains on $S$. mutans count as well as salivary $\mathrm{pH}$ and buffer capacity.

Streptococcus salivarius is a predominant human commensal of the oropharynx and can constitute a large proportion of the total bacterial population inhabiting this region. ${ }^{22}$ Burton et al. ${ }^{23}$ have demonstrated the beneficial effect of $S$ salivarius $\mathrm{K} 12$ and also highlighted the fact that this agent is well tolerated when regularly ingested in large numbers for 28 days. Salivarius K12 was first isolated from saliva of health child and being used in New Zealand for more than a decade, ${ }^{24} \mathrm{~K} 12$ produces salivaricin $\mathrm{A} 2$ and salivaricin B, two bacteriocins that effectively inhibit phylogenetically related bacterial species. In the human population, approximately $2 \%$ of children naturally possess salivarius strains that produce both salivaricin A and salivaricin B, which corresponds to the strain $\mathrm{K} 12$ bacteriocin profile. Due to its bacteriocin profile, BLIS K12 ${ }^{\mathrm{TM}}$ was commercially developed and thus became the first Commercial $\mathrm{K} 12$ based oral probiotic to specifically target the oral tissues. Hence in our clinical trial one group of patients received BLIS $\mathrm{K} 12^{\mathrm{TM}}$.

Another Strain of interest of our study is salivarius M18. This strain exhibits a very different bacteriocin profile when compared to K12. It effectively secretes the bacteriocins A2, 9, MPS and M. Due to its ability to inhibit cariogenic pathogens, BLIS M18 ${ }^{\mathrm{TM}}$ utilizing an active strain of salivarius M18 was commercially developed. ${ }^{25}$ For our study, subjects enrolled in the second group received BLIS $\mathrm{M} 18^{\mathrm{TM}}$. In a study by Burton et $a l .{ }^{17}$, they have provided evidence to support the use of S.salivarius M18, a bacterium isolated from the human oral cavity and shown to have antibacterial activity against a number of clinically important human pathogens to reduce plaque accumulation in school children. Since there is a lack of clinical evidence in support of S. salivarius K12, the current study was embarked on to clinically compare the two strains K12 and M18. 
Though most studies on probiotics and dental caries have evaluated the changes in MS counts, but only a few studies have assessed the impact on the $\mathrm{pH}$ and buffer capacity thus altering the overall chance to develop new carious lesions. ${ }^{15}$ Hence this randomized double blinded clinical trial was undertaken to evaluate and compare the effect of probiotic Streptococcus salivarius K12 and M18 on the Streptococcus mutans count, salivary $\mathrm{pH}$ and buffer capacity. When K12 and M18 were first introduced in the oral cavity in the form of a probiotic lozenge, they were expected to colonize specific oral tissue and be tolerated by the human host. Studies have demonstrated that treatment dosing regimen of one lozenge per day, taken before bedtime was well tolerated by the individuals. $^{26}$

A study by Power et al. showed that a lozenge containing streptococcus strain demonstrated an $80 \%$ effectiveness in colonizing the oral cavity among those who consume the probiotic while the controls ingesting powdered form showed a $33 \%$ decrease in the effectiveness. This reduced efficacy of the powdered form was attributed to a reduced oral exposure time when compared to the lozenge form of probiotic strain. ${ }^{26}$ Thus the lozenge form of the Streptococcus salivarius $\mathrm{K} 12$ and M18 was used in the current trial.

Before salivarius $\mathrm{K} 12$ and M18 can exert their beneficial effects, they must individually colonize the oral cavity. To initiate their colonisation in the oral cavity, individual cells must adhere to oral epithelial cells and then rapidly reproduce to form colonies. ${ }^{27}$ According to our results, 30days treatment with both BLIS $\mathrm{K} 12^{\mathrm{TM}}$ and BLIS M18 ${ }^{\mathrm{TM}}$ have shown reduction in the $S$. mutans count. This may be attributed to the ability of the salivarius K12 and M18 to colonize the oral tissues. Studies have shown that the higher levels of colonisation result in greater reduction of these caries causing bacteria in saliva and thus an overall reduction in the development of caries. ${ }^{28}$ However, studies have also shown that the patterns of colonisation of the oral cavity by S. salivarius $\mathrm{K} 12$ and M18 are dose dependent. ${ }^{27}$ When larger dosages are administered, an increased number of M18 bacteria are retained over longer periods of time. Thus further long term clinical trials comparing the two stains of the Streptococcus salivarius have to be undertaken to establish the dosage.

The results of the present trial have also demonstrated an increase in the salivary $\mathrm{pH}$ following a 30-day use of BLIS K12 $2^{\mathrm{TM}}$ and BLIS $\mathrm{M} 18^{\mathrm{TM}}$. Our results are in concordance with the results of Pierro et al.(15). Several microbial species belonging to the oral microbiota produce active urease as demonstrated by the Streptococcus salivarius and the urease from these species facilitates hydrolysis of urea. ${ }^{29}$ This enzyme urease can increase the $\mathrm{pH}$ of saliva by breaking down urea into carbon dioxide and ammonia, thus increasing the salivary $\mathrm{pH}$ thereby preventing hydroxyapatite dissolution. ${ }^{15}$ This could be an attributable reason for the increase in salivary $\mathrm{pH}$ following a 30-day use of both the probiotic strains. Though there was difference in the $S$. mutans count and salivary $\mathrm{pH}$, the buffer capacity of saliva remained unaltered in all three groups. This shows that there is no impact of these probiotics on the buffer capacity of saliva.

To improve the validity and accuracy of the conclusion drawn from the research, more extensive research with larger sample size should be conducted. In the field of probiotic research, there is always a continuous demand for more information about this relatively novel branch of probiotics.

\section{CONCLUSIONS}

Within the limitations of the present study it can be concluded that a 30day use of Streptococcus salivarius $\mathrm{K} 12$ and M18 resulted in a reduction in the Streptococcus mutans count while simultaneously improving the salivary $\mathrm{pH}$. Thus it is very evident that the use of probiotic Streptococcus salivarius K12 and M18 for a short period will definitely provide anticariogenic benefits. With the ever changing scenario in the field of caries prevention, further trials are needed to establish the long term effects of these probiotics in terms of practical clinical application. 


\section{COMPLIANCE WITH ETHICAL STANDARDS}

The authors declared that the paper has complied with the ethical standards as per the guidelines of the journal.

\section{CONFLICT OF INTEREST}

First author Dr Saravanan Poorni declares that she has no conflict of interest. Second Author Dr Nivedhitha M S declares that he has no conflict of interest. Third author Dr Manali Ramakrishnan Srinivasan declares that she has no conflict of interest.

\section{ACKNOWLEDGE}

I thank Dr Anusha, Senior Lecturer, Department of Public Health Dentisty, Ragas Dental College and Hospital, for her support in statistical analysis.

\section{REFERENCES}

1. Paster BJ, Boches SK, Galvin JL, Ericson RE, Lau $\mathrm{CN}$, Levanos VA, et al. Bacterial Diversity in Human Subgingival Plaque [Internet]. Vol. 183, Journal of Bacteriology. 2001. p. 3770-3783.

2. Paster BJ, Olsen I, Aas JA, Dewhirst FE. The breadth of bacterial diversity in the human periodontal pocket and other oral sites. Periodontol 2000. 2006;42:80-7.

3. Aas JA, Paster BJ, Stokes LN, Olsen I, Dewhirst FE. Defining the normal bacterial flora of the oral cavity. J Clin Microbiol. 2005 Nov;43(11):5721-5732.

4. Bowen WH, Koo H. Biology of Streptococcus mutans-derived glucosyltransferases: role in extracellular matrix formation of cariogenic biofilms. Caries Res. 2011 Feb 23;45(1):69-86.

5. Marsh PD. Are dental diseases examples of ecological catastrophes? [Internet]. Vol. 149, Microbiology. 2003. p. 279-294.

6. Paes Leme AF, Koo H, Bellato CM, Bedi G, Cury JA. The role of sucrose in cariogenic dental biofilm formation--new insight. J Dent Res. 2006 Oct;:85(10):878-887.

7. Takahashi N, Nyvad B. The Role of Bacteria in the Caries Process [Internet]. Vol. 90, Journal of Dental Research. 2011. p. 294-303.
8. Marsh PD. Dental plaque as a biofilm and a microbial community - implications for health and disease [Internet]. Vol. 6, BMC Oral Health. 2006.

9. Meurman JH. Probiotics: do they have a role in oral medicine and dentistry? Eur J Oral Sci. 2005 Jun;113(3):188-196.

10. Russell DA, Ross RP, Fitzgerald GF, Stanton C. Metabolic activities and probiotic potential of bifidobacteria. Int J Food Microbiol. 2011 Sep 1;149(1):88-105.

11. Twetman S, Keller MK. Probiotics for Caries Prevention and Control [Internet]. Vol. 24, Advances in Dental Research. 2012. p. 98-102.

12. Jäsberg $H$, Söderling $E$, Endo A, Beighton $D$, Haukioja A. Bifidobacteria inhibit the growth of Porphyromonas gingivalis but not of Streptococcus mutans in an in vitro biofilm model. Eur J Oral Sci. 2016 Jun;124(3):251-258.

13. Sañudo AI, Luque R, Díaz-Ropero MP, Fonollá J, Bañuelos Ó. In vitro and in vivo anti-microbial activity evaluation of inactivated cells of Lactobacillus salivarius CECT 5713 against Streptococcus mutans. Arch Oral Biol. 2017 Dec;84:58-63.

14. Vuotto C, Longo F, Donelli G. Probiotics to counteract biofilm-associated infections: promising and conflicting data. Int J Oral Sci. 2014 Dec;6(4):189194.

15. Pierro FD, Di Pierro F, Zanvit A, Nobili P, Risso P, Fornaini C. Cariogram outcome after 90 days of oral treatment with Streptococcus salivarius M18 in children at high risk for dental caries: results of a randomized, controlled study [Internet]. Clinical, Cosmetic and Investigational Dentistry. 2015. p. 107.

16. Hedayati-Hajikand T, Lundberg U, Eldh C, Twetman S. Effect of probiotic chewing tablets on early childhood caries--a randomized controlled trial. BMC Oral Health. 2015 Sep 24;15(1):112.

17. Burton JP, Drummond BK, Chilcott CN, Tagg JR, Thomson WM, Hale JDF, et al. Influence of the probiotic Streptococcus salivarius strain M18 on indices of dental health in children: a randomized double-blind, placebo-controlled trial. J Med Microbiol. 2013 Jun;62(Pt 6):875-884. 
18. Loesche WJ. Role of Streptococcus mutans in human dental decay. Microbiol Rev. 1986 Dec;50(4):353-380.

19. Kleinberg I. A mixed-bacteria ecological approach to understanding the role of the oral bacteria in dental caries causation: an alternative to Streptococcus mutans and the specific-plaque hypothesis. Crit Rev Oral Biol Med. 2002;13(2):108-125.

20. Hata S, Mayanagi H. Acid diffusion through extracellular polysaccharides produced by various mutants of Streptococcus mutans. Arch Oral Biol. 2003 Jun;48(6):431-438.

21. Green GE. Inherent Defense Mechanisms in Saliva [Internet]. Vol. 45, Journal of Dental Research. 1966. p. 624-629.

22. Könönen E, Jousimies-Somer H, Bryk A, Kilpi T, Kilian M. Establishment of streptococci in the upper respiratory tract: longitudinal changes in the mouth and nasopharynx up to 2 years of age. J Med Microbiol. 2002 Sep;51(9):723-730.

23. Burton JP, Cowley S, Simon RR, McKinney J, Wescombe PA, Tagg JR. Evaluation of safety and human tolerance of the oral probiotic Streptococcus salivarius K12: a randomized, placebo-controlled, double-blind study. Food Chem Toxicol. 2011 Sep;49(9):2356-2364.

24. Burton JP, Wescombe PA, Moore CJ, Chilcott CN, Tagg JR. Safety assessment of the oral cavity probiotic
Streptococcus salivarius K12. Appl Environ Microbiol. 2006 Apr;72(4):3050-3053.

25. Wescombe PA, Hale JDF, Heng NCK, Tagg JR. Developing oral probiotics fromStreptococcus salivarius [Internet]. Vol. 7, Future Microbiology. 2012. p. 1355-1371.

26. Power DA, Burton JP, Chilcott CN, Dawes PJ, Tagg JR. Preliminary investigations of the colonisation of upper respiratory tract tissues of infants using a paediatric formulation of the oral probiotic Streptococcus salivarius K12. Eur J Clin Microbiol Infect Dis. 2008 Dec;27(12):1261-1263.

27. Burton JP, Wescombe PA, Macklaim JM, Chai MHC, Macdonald K, Hale JDF, et al. Persistence of the oral probiotic Streptococcus salivarius M18 is dose dependent and megaplasmid transfer can augment their bacteriocin production and adhesion characteristics. PLoS One. 2013 Jun 13;8(6):e65991.

28. Burton JP, Chilcott CN, Moore CJ, Speiser G, Tagg JR. A preliminary study of the effect of probiotic Streptococcus salivarius $\mathrm{K} 12$ on oral malodour parameters [Internet]. Vol. 100, Journal of Applied Microbiology. 2006. p. 754-764.

29. Chen Y-YM, Burne RA. Identification and Characterization of the Nickel Uptake System for Urease Biogenesis in Streptococcus salivarius 57.I [Internet]. Vol. 185, Journal of Bacteriology. 2003. p. 6773-6779. 